Supporting information for

\title{
Efficient energy funnelling by engineering the bandgap of a perovskite: Förster resonance energy transfer or charge transfer?
}

Zhixing Gan, ${ }^{1,3 *}$ Weijian Chen, ${ }^{2}$ Chunhua Zhou, ${ }^{2}$ Liyan Yu, ${ }^{3}$ Lifeng Dong, ${ }^{3}$ Baohua $\mathrm{Jia}^{2 *}$ and Xiaoming $\mathrm{Wen}^{2 *}$

1 Jiangsu Key Lab on Opto-Electronic Technology, School of Physics and Technology, Nanjing Normal University, Nanjing 210023, China 2 Centre for Translational Atomaterials, Faculty of Science, Engineering and Technology, Swinburne University of Technology, John Street, Hawthorn, VIC 3122, Australia.

3 College of Materials Science and Engineering, Qingdao University of Science and Technology, Qingdao 266042, P. R. China.

*E-mail: zxgan@njnu.edu.cn, bjia@swin.edu.au, xwen@swin.edu.au 
(a)

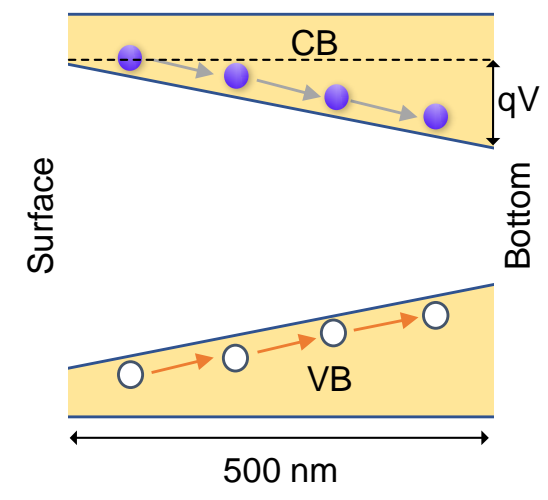

(b)

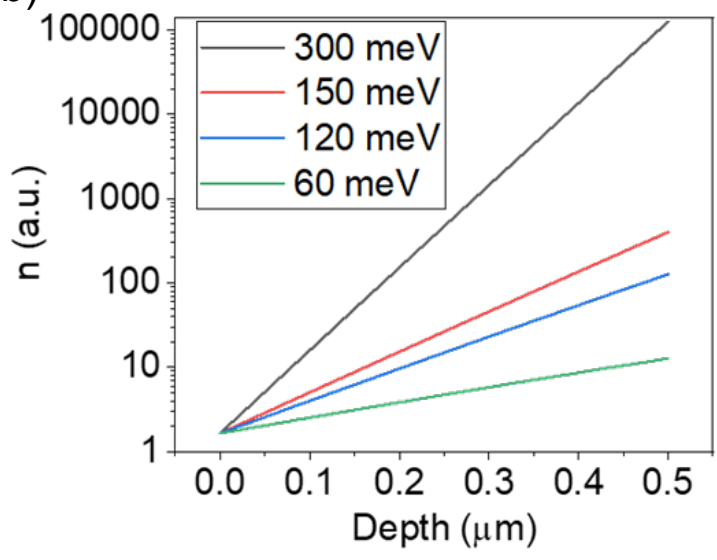

Figure S1. (a) Scheme of a graded bandgap. The electric potential at surface is higher than the bottom with a difference of qV. (b) Calculated carrier distributions when the MP with a graded bandgap is excited by a CW laser under CT model. The slopes are changed by adjusting $q V$ from 60 to $300 \mathrm{meV}$.

Different to the external electric field applied on a uniform semiconductor that resulting in opposite drift directions of electrons and holes, gradient bandgap drives both electrons and holes to move directionally from large bandgap to low bandgap. Taking the electron distribution as an example, regarding a sloping conduction band (CB), the electrons concentrate to the bottom due to the carrier mobility. After reaching an equilibrium state, the carrier density becomes $n(z)=n_{0}+\tau g(1-$ $\left.\frac{s \tau}{L_{D}+s \tau} e^{-\frac{H-z}{L_{D}}}\right) \exp \left(\frac{q V(z)}{k T}\right) \cdot q V(z)$ is the distribution of the electric potential in a graded bandgap. Assuming a simple linear slope, the calculated electron distributions of a graded bandgap are shown in Figure S1b. The carriers mainly distribute at the bottom due to the funnelling effect. Similar conclusion also can be acquired for hole distributions. 
(a)
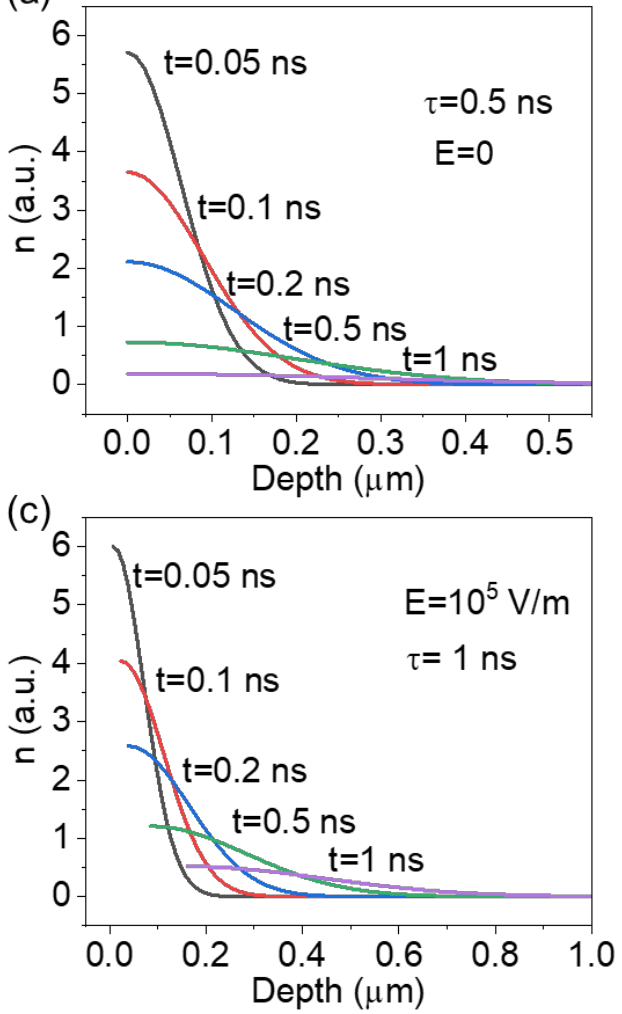

(b)
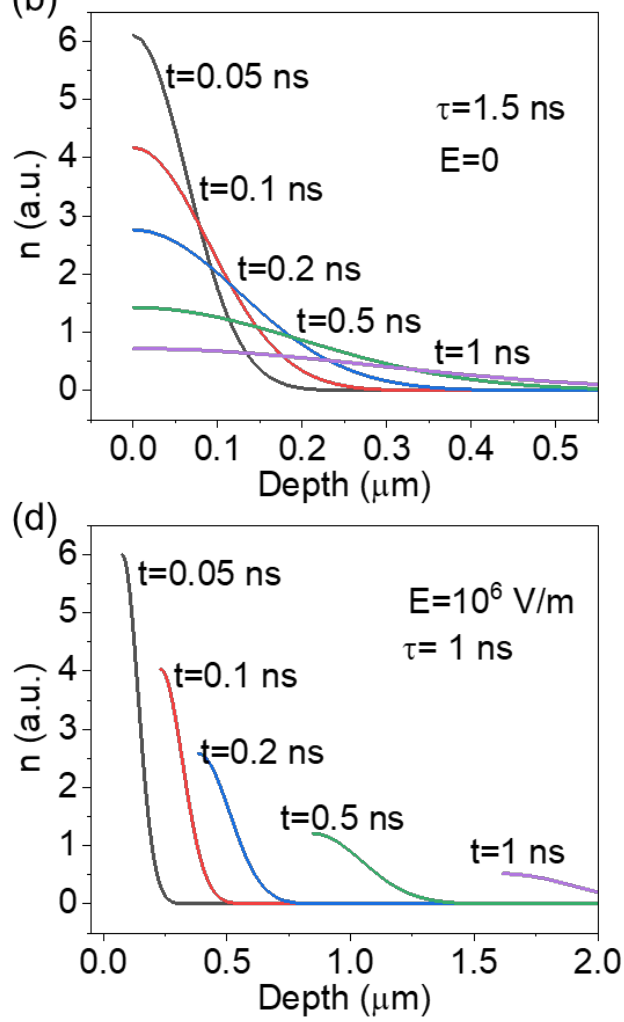

Figure S2. Calculated carrier distributions when the MP is excited by a pulsed laser under CT model. (a, b) Assuming carrier lifetime is $0.5 \mathrm{~ns}$ (a) and $1.5 \mathrm{~ns}$ (b) without electric field. (c, d) Assuming electric field is $10^{5} \mathrm{~V} / \mathrm{m}$ (c) and $10^{6} \mathrm{~V} / \mathrm{m}$ (d) when carrier lifetime is $1 \mathrm{~ns}$.

As shown in Figure S2a, when carrier lifetime is 0.5 ns, the carrier distribution boundary decreases to $0.4 \mu \mathrm{m}$. Whereas, if carrier lifetime increases to $1.5 \mathrm{~ns}$, the carrier distribution range increases accordingly (Figure S2b). Considering the electric field $E$ induced by the graded bandgap $E=q V / H$, the carrier mobility is included by replacing the $z$ to $z-\mu E t$, where $\mu$ is the carrier mobility coefficient $\frac{D}{\mu}=\frac{k T}{q}$. Thus, the equation (6) is changed to $n(t, z)=\frac{\Delta N_{0}}{\sqrt{4 \pi D t}} \exp \left[-\left(\frac{z-\mu E t^{2}}{4 D t}+\frac{t}{\tau}\right)\right]$. Assuming the $E$ is $10^{5} \mathrm{~V} / \mathrm{m}$ and carrier lifetime is $1 \mathrm{~ns}$, the carrier distributions at different decay times $(t=0.05,0.1,0.2$ 0.5, $1 \mathrm{~ns})$ are plotted in Figure S2c. Compared to the results shown in Figure 2e, the carrier distribution depth evidently increases. At $t=1 \mathrm{~ns}$, the carriers mainly distribute in the range of $(0.2,0.7 \mu \mathrm{m})$, When the electric filed $E$ increases to 
$10^{6} \mathrm{~V} / \mathrm{m}$, the carrier distribution depth further increases significantly. At $t=1 \mathrm{~ns}$, the carriers can penetrate to a depth of $2 \mu \mathrm{m}$.

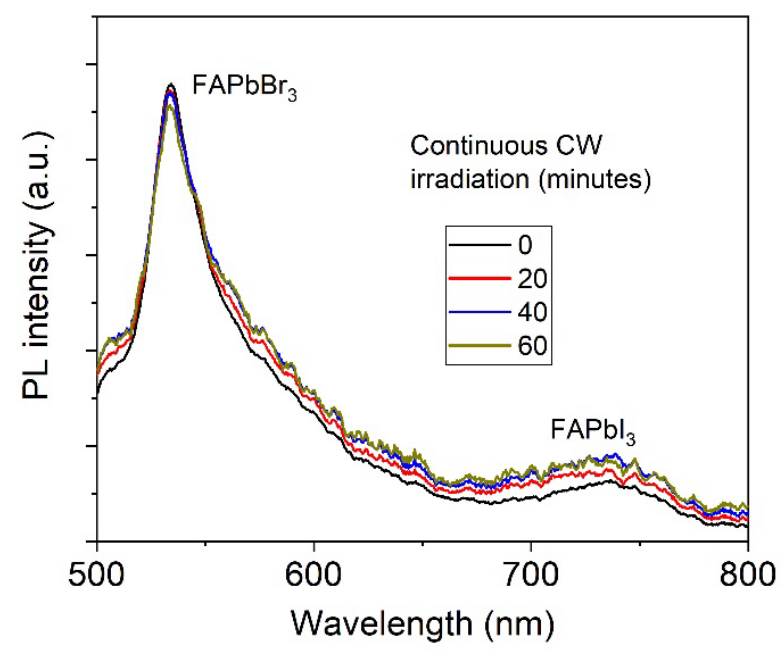

Figure S3. Photostability of the $\mathrm{FAPbBr}_{3} / \mathrm{FAPb}\left(\mathrm{Br}_{\mathrm{x}} \mathrm{I}_{1-\mathrm{x}}\right)_{3} / \mathrm{FAPbI}_{3}$ composite MPs. A strong CW laser $\left(0.443 \mathrm{KW} / \mathrm{cm}^{2}\right)$ is continuously irradiated on the composite MPs. And PL spectra are measured at a time interval of 20 minutes. The PL intensity only shows a very tiny decrease after one-hour irradiation, indicating the composite MP is stable under the measurement conditions.

\section{Discussion on reversibility of the $730 \mathrm{~nm}$-band}

In terms of the PL band emerging at $730 \mathrm{~nm}$ induced by CW laser, a similar case was also reported by Yang et al.[Organ. Electron. 34, 79-83 (2016)] The PL peak of $\mathrm{CH}_{3} \mathrm{NH}_{3} \mathrm{PbI}_{1.3} \mathrm{Br}_{1.7}$ film shifts from $\sim 640 \mathrm{~nm}$ to $\sim 750 \mathrm{~nm}$ when excited by a CW or a pulsed laser with high repetition rate, while keeps at $640 \mathrm{~nm}$ excited by pulsed laser with low repetition rate $(<500 \mathrm{~Hz})$. They proposed the redshift of PL was ascribed to the illumination induced sub-bandgap states. This illumination induced metastable states only emits when those states interact with more coming photons within their lifetimes. Thus, the $\sim 750 \mathrm{~nm}$ band only appears under excitation of a CW or a high repetition pulsed laser. It should be noted that the light induced modification of PL only 
can be recovered after keeping the film in dark for several hours at room temperature. In order to consolidate that the PL observations here are explained by the CT model rather than the illumination induced sub-bandgap states, the reversibility of the $730 \mathrm{~nm}$ band is tested by alternately exciting the thick composite MP without time interval. As displayed in Figure 5a, the 730 nm-band immediately recovers when the excitation is changed from pulsed to CW laser. Storing in dark for structural repair is not needed. Thus, the $730 \mathrm{~nm}$-band observed in our experiment cannot be caused by the light induced metastable state.

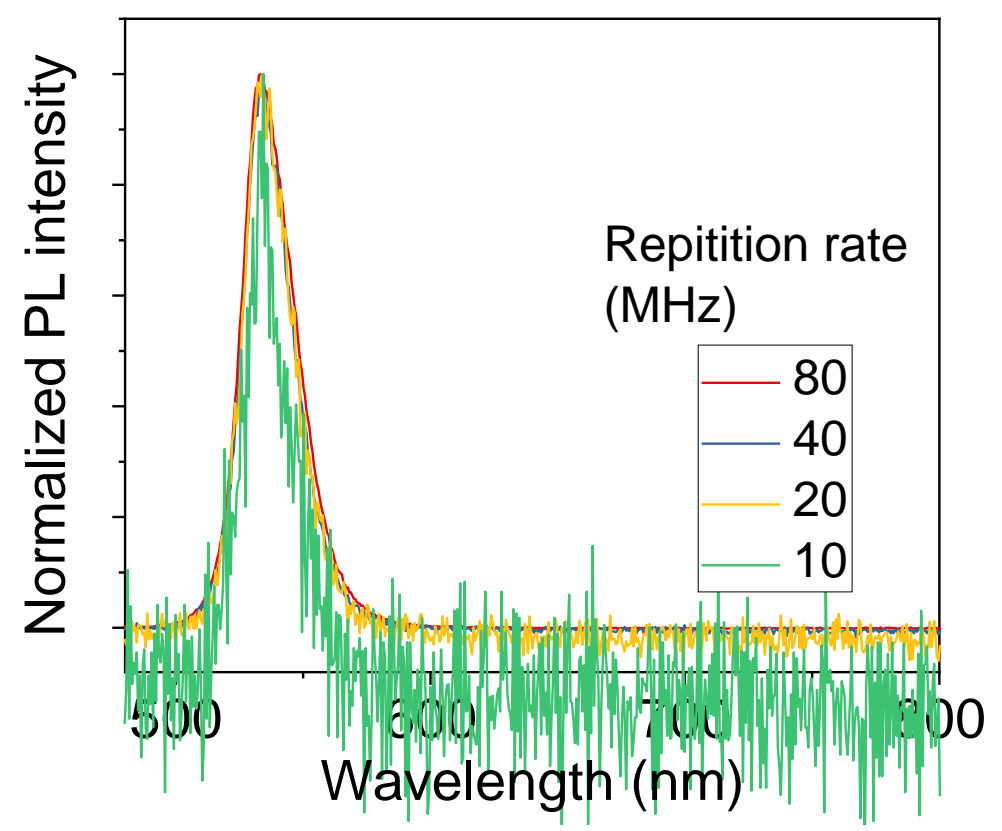

Figure S4. Normalized PL spectra of a thick composite MP excited by a pulsed laser with different repetition rates. The measurements conditions were kept the same by solely tuning the laser repetition rate. The PL spectra are almost the same except for different noises. 


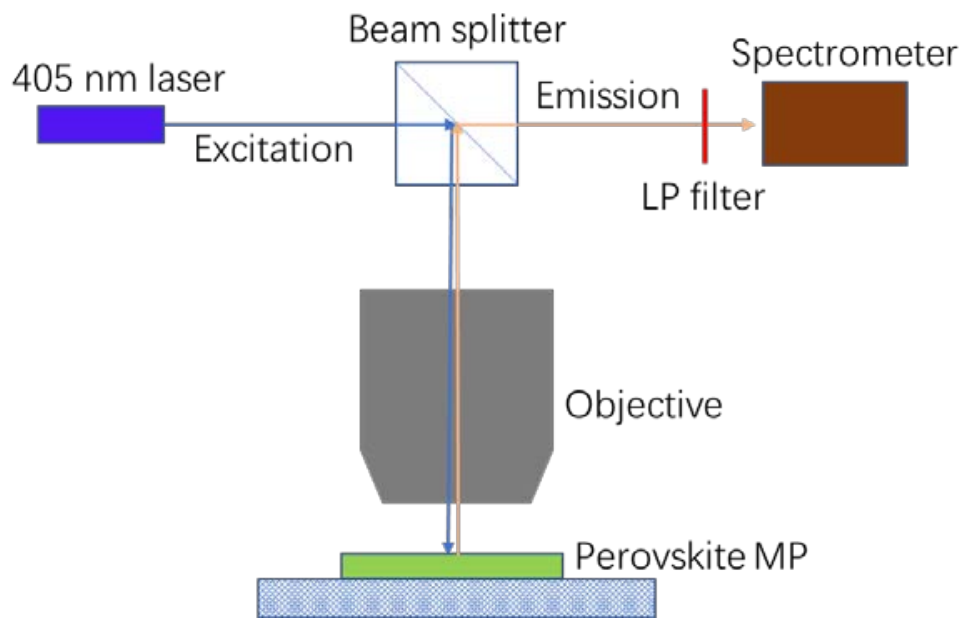

Figure S5. Experimental setup for PL experiments via a reflection model. All the PL results shown in this work are measured by this setup except for Figure S6b. The fluorescent signal is collected from the surface of the perovskite MP. LP filter is 430 nm long-pass filter.

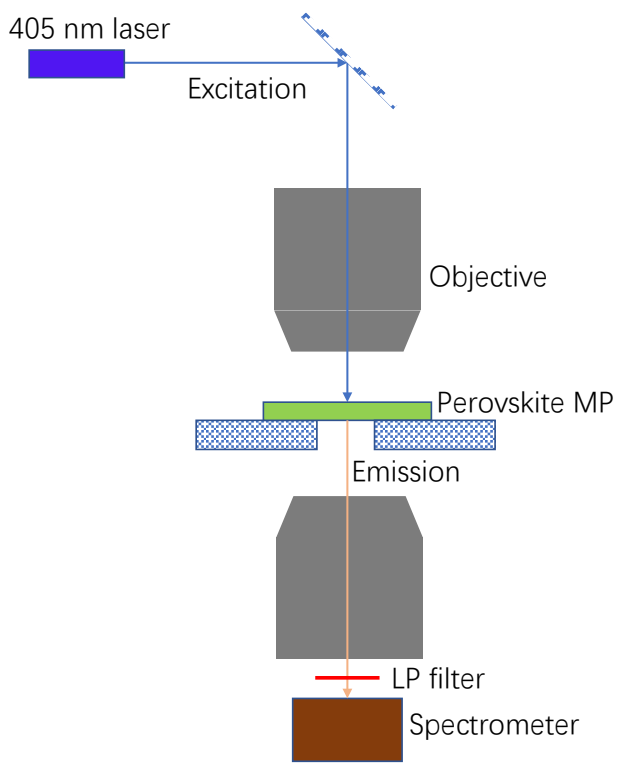

(b)

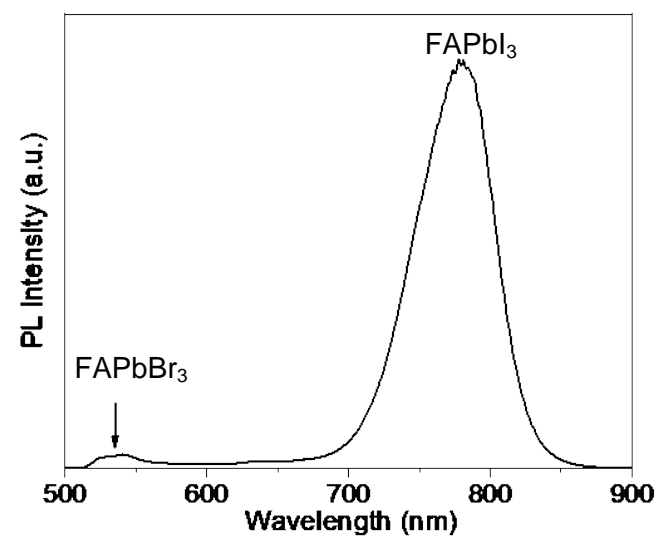

Figure S6. (a) Experimental setup for PL experiments via a transmission model. The fluorescent signal is collected from the bottom of the perovskite MP. LP filter is 430 nm long-pass filter. (b) PL spectrum of a thick $\mathrm{FAPbBr} 3 / \mathrm{FAPb}\left(\mathrm{Br}_{\mathrm{x}} \mathrm{I}_{1-\mathrm{x}}\right)_{3} / \mathrm{FAPbI}_{3}$ sandwich MP. The $530 \mathrm{~nm}$ emission of the surface $\mathrm{FAPbBr}$ is reabsorbed by the bottom $\mathrm{FAPbI}_{3}$, thus the $530 \mathrm{~nm}$ emission is relatively weak in this situation. 